\title{
Management of Graves' hyperthyroidism and orbitopathy in time of COVID-19 pandemic
}

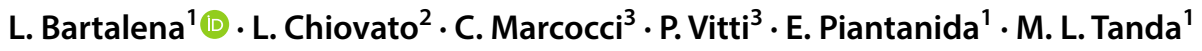

Received: 29 April 2020 / Accepted: 6 May 2020 / Published online: 21 May 2020

(C) Italian Society of Endocrinology (SIE) 2020

Keywords Graves' disease · Graves' orbitopathy · Antithyroid drugs · Glucocorticoids · COVID-19

\section{Introduction}

The Coronavirus disease 2019 (COVID-19) pandemic has prompted reflections and statements on the management of endocrine and metabolic disorders, including diabetes mellitus, obesity, adrenal insufficiency [1,2], as well as on the way COVID-19 outbreak might affect our daily clinical practice after the pandemic will be over [3].

Graves' disease is by far the most common cause of hyperthyroidism, with a $>1 \%$ prevalence in the general population [4]. Graves' orbitopathy (GO) is its most frequent extrathyroidal expression, affecting about $25-30 \%$ of Graves' patients and being severe enough to require active immunosuppressive treatments in 5\% of them [5]. Antithyroid drugs (ATDs), mainly methimazole (MMI), less frequently propylthiouracil (PTU), represent the first-line treatment for Graves' hyperthyroidism worldwide [6], but frequent relapses after ATD withdrawal make it often unavoidable thyroid ablation by either radioactive iodine (RAI) or thyroidectomy [6] that cause permanent hypothyroidism.

While there is no current evidence that Graves' disease per se or ATD treatment increase the risk of COVID-19 infection, a Graves' patient might be more prone to develop symptomatic forms of COVID-19 infection. Uncontrolled

L. Bartalena

luigi.bartalena@uninsubria.it;

luigi.bartalena@asst-settelaghi.it

1 Endocrine Unit, Department of Medicine and Surgery, University of Insubria, ASST dei Sette Laghi, Viale Borri, 57, 21100 Varese, Italy

2 Unit of Internal Medicine and Endocrinology, Laboratory for Endocrine Disruptors, Istituti Clinici Scientifici Maugeri IRCCS, Pavia, Italy

3 Department of Clinical and Experimental Medicine, University of Pisa, Pisa, Italy hyperthyroidism is associated in the elderly to cardiovascular diseases, that is the main cause of Graves' patients excess mortality [7, 8], but also a cause of COVID-19-related mortality. On the other hand, infections, particularly respiratory infections, can precipitate thyroid storm, a life-threatening exacerbation of untreated/poorly treated or decompensated hyperthyroidism, characterized by multiorgan failure [9]. Thus, COVID-19 infection is a risk factor for a poor control of hyperthyroidism, which, in turn, may contribute to the infection-related mortality risk. The logical consequence is that patients with Graves' hyperthyroidism, particularly the elderly, should be given particular attention by general practitioners and thyroid specialists.

\section{Treatment of non-COVID-19 Graves' patients}

ATDs remain the treatment of choice under these conditions. Although patients are normally treated for 18-24 months, there is evidence that long-term ATD treatment is safe, particularly if low doses of the drug are required to control thyroid hormone excess [10]. In addition, during the pandemic outburst, non-urgent surgery, as well as RAI treatment, have been stopped. Therefore, unless there is an emergency (hyperthyroidism refractory to ATDs, very large goiters with severe compressive symptoms), definitive treatments should be postponed.

After the initial control of thyroid hyperfunction with appropriate dosage of the drug, ATDs can be given by the titration regimen (using the lowest dose of the drug that maintains euthyroidism) or the block-and-replace regimen (using constantly high doses of MMI in association with levothyroxine to avoid hypothyroidism) [6]. The latter method may have the advantage, during the pandemic, to avoid repeated thyroid function testing and visits. The drawback of this strategy, mainly in patients managed by telemedicine, 
is an increased risk of adverse events due to the use of high doses of ATDs. In this regard, agranulocytosis deserves particular attention. This is a rare, but life-threatening complication, affecting probably more patients than previously believed (up to $1.2 \%$ ), particularly in the initial phases of MMI treatment, when higher doses of the drug are used [11], and in the elderly [12]. Because infection symptoms (fever, sore throat, flu-like symptoms) due to agranulocytosis may overlap with and be difficult to distinguish from those of COVID-19 infection, MMI should be stopped and an urgent white blood cell count should be obtained. If agranulocytosis is ruled out, but low lymphocyte counts are discovered, ATDs can be continued, but testing for COVID-19 should be considered. ATD treatment should then be continued until either RAI treatment or thyroidectomy, if deemed necessary, is feasible.

\section{Treatment of patients with Graves' hyperthyroidism and COVID-19 infection}

Any hyperthyroid patient with COVID-19 infection should continue ATD treatment, irrespective of being hospitalized or followed in quarantine at home or in dedicated structures, because, as mentioned above, uncontrolled hyperthyroidism adds on COVID-19 infection to determine an unfavorable outcome. MMI and PTU are administered orally if the COVID-19 patient can eat, or by a nasogastric tube, if he/ she is unable to swallow. In extreme situations, alternative routes can be used. These include rectal administration by suppositories or water enema formulations: the enema form seems to be more effective than suppository tablets [13]. Intravenous preparations of MMI or PTU are not available in most countries, including Italy, but can be prepared by sterile pharmacies under aseptic techniques. MMI, being more soluble than PTU (and PTU is not available in several countries), can be dissolved in a $\mathrm{pH}$-neutral $0.9 \%$ sodium chloride solution, and given as a 2-min pulse followed by a saline flush [14].

\section{Management of Graves' orbitopathy in time of COVID-19}

Mild GO does not require active treatment with immunosuppressive drugs. Thus, the usual preventive/therapeutic actions (refrain from smoking, artificial tears and ointments, stable maintenance of euthyroidism) can be taken [15]. Selenium, if indicated, can also be used [15].

Patients with moderate-to-severe and active GO represent a major dilemma in this period. Intravenous glucocorticoids represent the first-line treatment [15], but high-dose oral glucocorticoids are also widely used, although less effective and less well tolerated [15]. Glucocorticoids cause immunosuppression, which is a risk factor for COVID-19 infection unfavorable outcome. On the other hand, severe, sight-threatening forms of GO do require aggressive glucocorticoid treatment to avoid irreversible loss of vision. There are no available guidelines for treating these patients in time of COVID-19. If GO is not very severe, systemic treatment should be postponed, and local treatments and preventive measures can be used [15]. In patients who are already under intravenous glucocorticoid treatment on a weekly basis, it might be conceivable to continue this treatment, provided that the hospital offers a clear separation and different paths between COVID-19 areas and COVID-free areas, and adequate shielding is given. Alternatively, and preferably to avoid unnecessary visits to the hospital, these patients might be shifted to oral glucocorticoid therapy, at home. It is essential to strongly recommend following general measures of social confinement and distancing, hands and surfaces hygiene, shielding if and when going outside. Although intravenous glucocorticoid therapy for GO does not seem to be associated with secondary adrenal insufficiency [16], patients who recently finished the intravenous glucocorticoid treatment, and more obviously, those who completed a long-term course of oral glucocorticoids for GO, should be considered at risk of potentially having an acute adrenal crisis if they get infected by Coronavirus. Accordingly, they should receive instructions on how to recognize symptoms of incipient adrenal insufficiency, inform immediately their family doctor, and adjust the daily dose of hydrocortisone as indicated for sick days.

Orbital decompression should be deferred, unless there is an immediate threat for the patient's sight. Likewise, rehabilitative surgery (squint surgery, eyelid surgery) should also be postponed.

\section{Compliance with ethical standards}

Conflict of interest The authors declare they have no conflict of interest.

Ethical approval This article is an Opinion paper, with no original experimental or clinical data, and thus no ethical approval is needed.

Informed consent Informed consent is not needed, because this article does not contain any original research on either animals or human subjects.

\section{References}

1. Puig-Domingo M, Marazuela M, Giustina A (2020) COVID-19 and endocrine diseases. A statement from the European Society of Endocrinology. Eur J Endocrinol 68(1):2-5. https://doi. org/10.1007/s12020-020-02294-5 
2. Isidori AM, Arnaldi G, Boscaro M, Falorni A, Giordano C, Giordano R, Pivonello R, Pofi R, Hasenmajer V, Venneri MA, Sbardella E, Simeoli C, Scaroni C, Lenzi A (2020) COVID-19 infection and glucocorticoids: update from the Italian Society of Endocrinology Expert Opinion in steroid replacement in adrenal insufficiency. J Endocrinol Invest. 2020 April 25 [Online ahead of print] https://doi.org/10.1007/s40618-020-01266-w

3. Trimarchi $F(2020)$ An invisible hand has guided clinical endocrinology toward an unexpected future. J Endocrinol Invest. 2020 April 20 [Online ahead of print] https://doi.org/10.1007/s4061 8-020-01256-y

4. Smith TJ, Hegedus L (2016) Graves' disease. N Engl J Med 375(16):1552-1565. https://doi.org/10.1056/nejmra1510030

5. Masiello E, Veronesi G, Gallo D, Premoli P, Bianconi E, Rosetti S, Cusini C, Sabatino J, Ippolito S, Piantanida E, Tanda ML, Chiovato L, Wiersinga WM, Bartalena L (2018) Antithyroid drug treatment for Graves' disease: baseline predictive models of relapse after treatment for a patient-tailored management. J Endocrinol Invest 41(12):1425-1432. https://doi.org/10.1007/ s40618-018-0918-9

6. Kahaly GJ, Bartalena L, Hegedus L, Leenhardt L, Poppe K, Pearce SH (2018) 2018 European Thyroid Association guideline for the management of Graves' hyperthyroidism. Eur Thyroid J 7(4):167186. https://doi.org/10.1159/000490384

7. Lillevang-Johansen M, Abrahamsen B, Jorgensen HL, Brix TB, Hegedus L (2017) Excess mortality in treated and untreated hyperthyroidism is related to cumulative periods of low serum TSH. J Clin Endocrinol Metab 102(7):2301-2309. https://doi. org/10.1210/jc.2017-00166

8. Okosieme OE, Taylor PN, Evans C, Thayer D, Chai A, Khan D, Draman MS, Tennant B, Green J, Sayers A, French R, Lazarus JH, Premawardhana LD, Dayan CM (2019) Primary therapy of Graves' disease and cardiovascular morbidity and mortality: a linked-record cohort study. Lancet Diabetes Endocrinol 7(4):278287. https://doi.org/10.1016/s2213-8587(19)30059-2
9. Akamizu T (2018) Thyroid storm: a Japanese perspective. Thyroid 28(1):32-40. https://doi.org/10.1089/thy.2017.0243

10. Azizi F, Malbbosbaf R (2019) Safety of long-term antithyroid drug treatment? A systematic review. J Endocrinol Invest 42(11):1273-1283. https://doi.org/10.1007/s40618-019-01054-1

11. Bartalena L, Chiovato L, Vitti P (2016) Management of hyperthyroidism due to Graves' disease: frequently asked questions and answers (if any). J Endocrinol Invest 39(10):1105-1114. https:// doi.org/10.1007/s40618-016-0505-x

12. Bukhari S, Khan M, Kumar N, Mohan V (2017) Increased risk for thionamide-induced agranulocytosis in elderly patients: a case presentation and literature review. BMJ Case Rep. https:// doi.org/10.1136/bcr-2017-220924

13. Alfadhli E, Gianoukakis AG (2011) Management of severe thyrotoxicosis when the gastrointestinal tract is compromised. Thyroid 21(3):215-220. https://doi.org/10.1089/thy.2010.0159

14. Hodak SP, Huang C, Clarke D, Burman KD, Jonklaas J, JanicicKharic N (2006) Intravenous methimazole in the treatment of refractory hyperthyroidism. Thyroid 16(7):291-295. https://doi. org/10.1089/thy.2006.16.691

15. Bartalena L, Baldeschi L, Boboridis K, Eckstein A, Kahaly GJ, Marcocci C, Perros P, Salvi M, Wiersinga WM (2016) The 2016 European Thyroid Association/European Group on Graves' Orbitopathy guidelines for the management of Graves' orbitopathy. Eur Thyroid J 5(1):9-26. https://doi.org/10.1159/000443828

16. Jespersen S, Nyygard B, Ostergaard Kristensen L (2015) Methylprednisolone pulse treatment of Graves' ophthalmopathy is not associated with secondary adrenocortical insufficiency. Eur Thyroid J 4(4):222-225. https://doi.org/10.1159/000440834

Publisher's Note Springer Nature remains neutral with regard to jurisdictional claims in published maps and institutional affiliations. 